PROCEEDINGS OF THE

AMERICAN MATHEMATICAL SOCIETY

Volume 135, Number 7, July 2007, Pages 2113-2120

S 0002-9939(07)08708-4

Article electronically published on February 28, 2007

\title{
ON THE HELTON CLASS OF $p$-HYPONORMAL OPERATORS
}

\author{
YOENHA KIM, EUNGIL KO, AND JI EUN LEE
}

(Communicated by Joseph A. Ball)

\begin{abstract}
In this paper we show that the Helton class of $p$-hyponormal operators has scalar extensions. As a corollary we get that each operator in the Helton class of $p$-hyponormal operators has a nontrivial invariant subspace if its spectrum has its interior in the plane.
\end{abstract}

\section{InTRODUCTION}

Let $H$ be a complex (separable) Hilbert space and let $\mathcal{L}(H)$ denote the algebra of all bounded linear operators on $H$. If $T \in \mathcal{L}(H)$, we write $\sigma(T)$ for the spectrum of $T$.

An operator $T \in \mathcal{L}(H)$ is said to be $p$-hyponormal, $0<p \leq 1$, if $\left(T^{*} T\right)^{p} \geq$ $\left(T T^{*}\right)^{p}$, where $T^{*}$ is the adjoint of $T$. If $p=1, T$ is called hyponormal and if $p=\frac{1}{2}, T$ is called semi-hyponormal. Semi-hyponormal operators were introduced by Xia (see [15]), and $p$-hyponormal operators for a general $p, 0<p<1$, have been studied by Aluthge. Any $p$-hyponormal operators are $q$-hyponormal if $q \leq p$ by Löwner's theorem (see [13]). But there are examples to show that the converse of the above statement is not true (see [2]).

In [8] J. W. Helton initiated the study of operators $T$ which satisfy an identity of the form

$$
T^{* m}-\left(\begin{array}{c}
m \\
1
\end{array}\right) T^{* m-1} T+\cdots+(-1)^{m} T^{m}=0 .
$$

We need further study for this class of operators based on (1). Let $R$ and $S$ be in $\mathcal{L}(H)$ and let $C(R, S): \mathcal{L}(H) \rightarrow \mathcal{L}(H)$ be defined by $C(R, S)(A)=R A-A S$. Then

$$
C(R, S)^{k}(I)=\sum_{j=0}^{k}(-1)^{k-j}\left(\begin{array}{c}
k \\
j
\end{array}\right) R^{j} S^{k-j} .
$$

Definition 1.1. Let $R \in \mathcal{L}(H)$. If there is an integer $k \geq 1$ such that an operator $S$ satisfies $C(R, S)^{k}(I)=0$, we say that $S$ belongs to the Helton class of $R$ with order $k$. We denote this by $S \in \operatorname{Helton}_{k}(R)$.

We remark that $C(R, S)^{k}(I)=0$ does not imply $C(S, R)^{k}(I)=0$ in general.

Received by the editors December 14, 2004 and, in revised form, March 13, 2006.

2000 Mathematics Subject Classification. Primary 47B20; Secondary 47A10.

This work was supported by a grant (R14-2003-006-01000-0) from the Korea Research Foundation.

(C)2007 American Mathematical Society Reverts to public domain 28 years from publication 
Example 1.2. Let $S$ and $R$ be operators in $\mathcal{L}(H \oplus H \oplus H)$ defined by the following:

$$
S=\left(\begin{array}{ccc}
0 & A & B \\
0 & 0 & 0 \\
0 & 0 & 0
\end{array}\right) \quad \text { and } \quad R=\left(\begin{array}{ccc}
0 & 0 & C \\
0 & 0 & D \\
0 & 0 & 0
\end{array}\right),
$$

where $A, B, C$, and $D$ are bounded linear operators defined on $H$. Then it is easy to calculate that $C(R, S)^{2}(I)=0$, but $C(S, R)^{2}(I) \neq 0$.

A bounded linear operator $S$ on $H$ is called scalar of order $m$ if it possesses a spectral distribution of order $m$, i.e., if there is a continuous unital morphism of topological algebras

$$
\Phi: C_{0}^{m}(C) \longrightarrow \mathcal{L}(H)
$$

such that $\Phi(z)=S$, where $z$ stands for the identity function on $C$, and $C_{0}^{m}(C)$ stands for the space of compactly supported functions on $C$, continuously differentiable of order $m, 0 \leq m \leq \infty$. An operator is subscalar if it is similar to the restriction of a scalar operator to an invariant subspace.

In this paper we show that the Helton class of $p$-hyponormal operators has scalar extensions. As a corollary we get that the Helton class of $p$-hyponormal operators has a nontrivial invariant subspace if its spectrum has its interior in the plane.

\section{Preliminaries}

Let $z$ be the coordinate in the complex plane $C$ and let $d \mu(z)$ denote the planar Lebesgue measure. Fix a complex (separable) Hilbert space $H$ and a bounded (connected) open subset $U$ of $C$. We shall denote by $L^{2}(U, H)$ the Hilbert space of measurable functions $f: U \rightarrow H$, such that

$$
\|f\|_{2, U}=\left(\int_{U}\|f(z)\|^{2} d \mu(z)\right)^{\frac{1}{2}}<\infty .
$$

The space of functions $f \in L^{2}(U, H)$ which are analytic functions in $U$ (i.e., $\bar{\partial} f=0)$ is defined by

$$
A^{2}(U, H)=L^{2}(U, H) \cap \mathcal{O}(U, H)
$$

where $\mathcal{O}(U, H)$ denotes the Fréchet space of $H$-valued analytic functions on $U$ with respect to uniform topology. $A^{2}(U, H)$ is called the Bergman space for $U$. Note that $A^{2}(U, H)$ is a Hilbert space. We denote by $P$ the orthogonal projection of $L^{2}(U, H)$ onto $A^{2}(U, H)$.

Let $T \in \mathcal{L}(H)$. The study of the operator $T-z$ on the space $\mathcal{O}(U, H)$ led E. Bishop to fundamental results in spectral theory. Among other things he isolated in [4] the single-valued extension property, which means by definition that the operator $T-z$ acts one-to-one on $\mathcal{O}(U, H)$ for an arbitrary open subset $U$ of $C$, and the property $(\beta)$, which requires that $T-z$ should be one-to-one and with closed range on $\mathcal{O}(U, H)$ for every open set $U$.

Let us define now a special Sobolev type space. Let $U$ be again a bounded open subset of $C$ and $m$ be a fixed nonnegative integer. The vector-valued Sobolev space $W^{m}(U, H)$ with respect to $\bar{\partial}$ and of order $m$ will be the space of those functions $f \in L^{2}(U, H)$ whose derivatives $\bar{\partial} f, \cdots, \bar{\partial}^{m} f$ in the sense of distributions still belong to $L^{2}(U, H)$. Endowed with the norm

$$
\|f\|_{W^{m}}^{2}=\sum_{i=0}^{m}\left\|\bar{\partial}^{i} f\right\|_{2, U}^{2}
$$


$W^{m}(U, H)$ becomes a Hilbert space contained continuously in $L^{2}(U, H)$.

Let $U$ be a (connected) bounded open subset of $C$ and let $m$ be a nonnegative integer. The linear operator $M$ of multiplication by $z$ on $W^{m}(U, H)$ is continuous and it has a spectral distribution of order $m$, defined by the functional calculus

$$
\Phi_{M}: C_{0}^{m}(C) \longrightarrow \mathcal{L}\left(W^{m}(U, H)\right), \Phi_{M}(f)=M_{f} .
$$

Therefore, $M$ is a scalar operator of order $m$.

\section{SOME PROperties}

Let $H$ be a complex (separable) Hilbert space with an orthonormal basis $\left\{e_{0}, e_{1}\right.$, $\cdots\}$ and let $S$ be the unilateral shift defined by $S e_{n}=e_{n+1}$ for all $n \geq 0$. If $\left\{\alpha_{n}\right\}_{n=0}^{\infty}$ is any bounded sequence of nonnegative numbers, a unilateral weighted shift $W$ with a weight sequence $\left\{\alpha_{n}\right\}$ is defined by $W e_{n}=\alpha_{n} e_{n+1}$ for $n \geq 0$.

Proposition 3.1. Let $S$ be the unilateral shift and let $W$ be a unilateral weighted shift with a weight sequence $\left\{\alpha_{n}\right\}_{n=0}^{\infty}$. Then $W \in \operatorname{Helton}_{k}(S)$ if and only if

$$
\sum_{j=0}^{k-1}(-1)^{k-j}\left(\begin{array}{c}
k \\
j
\end{array}\right) \alpha_{n} \cdots \alpha_{n+k-j-1}+1=0
$$

for $n \geq 0$.

Proof. From the definition, we know that $W \in \operatorname{Helton}_{k}(S)$ if and only if

$$
\sum_{j=0}^{k}(-1)^{k-j}\left(\begin{array}{c}
k \\
j
\end{array}\right) S^{j} W^{k-j}=0 .
$$

Since $S^{j} W^{k-j} e_{n}=\alpha_{n} \alpha_{n+1} \cdots \alpha_{n+k-j-1} e_{n+k}$ and $S^{k} e_{n}=e_{n+k}$ for $n \geq 0$, we get that $W \in \operatorname{Helton}_{k}(S)$ if and only if

$$
\sum_{j=0}^{k-1}(-1)^{k-j}\left(\begin{array}{c}
k \\
j
\end{array}\right) \alpha_{n} \cdots \alpha_{n+k-j-1}+1=0
$$

for $n \geq 0$.

The next corollary shows that Helton $2(S)$ contains non-p-hyponormal operators.

Corollary 3.2. Let $S$ be the unilateral shift and let $W$ be a unilateral weighted shift with a weight sequence $\left\{\alpha_{n}\right\}_{n=0}^{\infty}=\left\{2, \frac{3}{2}, \frac{4}{3}, \cdots, \frac{n+2}{n+1}, \cdots\right\}$ for $n \geq 1$. Then $W \in$ Helton $_{2}(S)$, but is not a p-hyponormal operator.

Proof. Since a weight sequence is decreasing, $W$ is not a $p$-hyponormal operator. Since $\alpha_{n} \alpha_{n+1}-2 \alpha_{n}+1=0$ for $n \geq 0$, from Proposition 3.1, $W \in \operatorname{Helton}_{2}(S)$.

Remark. $C(W, S)^{k}(I) \neq 0$ for all positive integers $k$, where $S$ is the unilateral shift and $W$ is the unilateral weighted shift in Corollary 3.2. Indeed, since

$$
W^{j} S^{k-j} e_{n}=\alpha_{n+k-j} \alpha_{n+k-j+1} \cdots \alpha_{n+k-1} e_{n+k}
$$


and $\alpha_{n}=\frac{n+2}{n+1}$ for all $n \geq 0$, we obtain

$$
\begin{aligned}
& 1+\sum_{j=1}^{k}(-1)^{k-j}\left(\begin{array}{c}
k \\
j
\end{array}\right) \alpha_{n+k-j} \cdots \alpha_{n+k-1} \\
= & 1+\sum_{j=1}^{k}(-1)^{k-j}\left(\begin{array}{c}
k \\
j
\end{array}\right) \frac{n+k+1}{n+k-j+1} \neq 0 .
\end{aligned}
$$

Hence, $C(W, S)^{k}(I) \neq 0$ for all positive integers $k$.

In general, when $R$ is a $p$-hyponormal operator, there exists a non-p-hyponormal operator $T$ which satisfies $C(R, T)^{k}(I)=0$, but $C(T, R)^{k}(I) \neq 0$ for some $k$.

Example 3.3. Let $S$ be a unilateral weighted shift with a weight sequence $\left\{\alpha_{n}\right\}_{n=0}^{\infty}$ $=\left\{\frac{1}{2}, \frac{2}{3}, \cdots\right\}$ and let $W$ be a unilateral weighted shift with a weight sequence $\left\{\beta_{n}\right\}_{n=0}^{\infty}=\left\{2, \frac{7}{6}, \frac{15}{14}, \frac{26}{25}, \cdots\right\}$ which satisfies $\beta_{n+1} \beta_{n}-\frac{2(n+2)}{n+3} \beta_{n}+\frac{n+1}{n+3}=0$. Then it is easy to show that $C(S, W)^{2}(I)=0$, but $C(W, S)^{2}(I) \neq 0$.

\section{SubSCALARITY}

In this section we consider the scalar extensions of the Helton class of $p$-hyponormal operators. We start this section with the definition of the Aluthge transform of an operator. An arbitrary operator $T \in \mathcal{L}(H)$ has a unique polar decomposition $T=U|T|$, where $|T|=\left(T^{*} T\right)^{\frac{1}{2}}$ and $U$ is the appropriate partial isometry satisfying $\operatorname{ker} U=\operatorname{ker}|T|$ and $\operatorname{ker} U^{*}=\operatorname{ker} T^{*}$. Associated with $T$ is a related operator $|T|^{\frac{1}{2}} U|T|^{\frac{1}{2}}$, called the Aluthge transform of $T$, and denoted throughout this paper by $\tilde{T}$. Lemma 4.1 is essential for the proof of our main theorem.

Lemma 4.1. Let $T \in \mathcal{L}(H)$ be a p-hyponormal operator where $0<p \leq 1$. If $\left\{f_{n}\right\}$ is a sequence in $W^{4 k}(D, H)$ such that $\lim _{n \rightarrow \infty}\left\|(T-z)^{k} \bar{\partial}^{i} f_{n}\right\|_{2, D}=0$ for $i=1, \cdots, 4 k$, then $\lim _{n \rightarrow \infty}\left\|(T-z) \bar{\partial}^{i} f_{n}\right\|_{2, D_{0}}=0$ for $i=1, \cdots, 4$ and $\lim _{n \rightarrow \infty}\left\|(I-P) f_{n}\right\|_{2, D_{0}}=$ 0 for every disk $D_{0}$ strictly contained in $D$.

Proof. Assume that $\left\{f_{n}\right\}$ is a sequence in $W^{4 k}(D, H)$ such that

$$
\lim _{n \rightarrow \infty}\left\|(T-z)^{k} \bar{\partial}^{i} f_{n}\right\|_{2, D}=0
$$

for $i=1, \cdots, 4 k$. Using the induction, we will show that

$$
\lim _{n \rightarrow \infty}\left\|(T-z)^{k-1} \bar{\partial}^{i} f_{n}\right\|_{2, D_{0}}=0
$$

for $i=1, \cdots, 4(k-1)$, where a disk $D_{0}$ is strictly contained in $D$. Since $|T|^{1 / 2} T=$ $\tilde{T}|T|^{1 / 2}$, we have

$$
\lim _{n \rightarrow \infty}\left\|(\tilde{T}-z)^{k}|T|^{\frac{1}{2}} \bar{\partial}^{i} f_{n}\right\|_{2, D}=0
$$

for $i=1, \cdots, 4 k$. Since $\tilde{T}$ is semihyponormal, by [10],

$$
\lim _{n \rightarrow \infty}\left\|(\tilde{T}-z)^{*}(\tilde{T}-z)^{k-1}|T|^{\frac{1}{2}} \bar{\partial}^{i} f_{n}\right\|_{2, D}=0
$$

for $i=1, \cdots, 4 k$. By an application of [14, Proposition 2.1], we get

$$
\lim _{n \rightarrow \infty}\left\|(I-P)(\tilde{T}-z)^{k-1}|T|^{\frac{1}{2}} \bar{\partial}^{i} f_{n}\right\|_{2, D}=0
$$


for $i=1, \cdots, 4 k-2$. From (4) and (5), we have

$$
\lim _{n \rightarrow \infty}\left\|(\tilde{T}-z) P(\tilde{T}-z)^{k-1}|T|^{\frac{1}{2}} \bar{\partial}^{i} f_{n}\right\|_{2, D}=0
$$

for $i=1, \cdots, 4 k-2$, where $P$ denotes the orthogonal projection of $L^{2}(D, H)$ onto the Bergman space $A^{2}(D, H)$. Since $\tilde{T}$ is semihyponormal, it satisfies the property ( $\beta$ ) from [11]. Hence

$$
\lim _{n \rightarrow \infty}\left\|P(\tilde{T}-z)^{k-1}|T|^{\frac{1}{2}} \bar{\partial}^{i} f_{n}\right\|_{2, D_{0}}=0
$$

for $i=1, \cdots, 4 k-2$, where a disk $D_{0}$ is strictly contained in $D$. Since $|T|^{1 / 2} T=$ $\tilde{T}|T|^{1 / 2}$, (6) and (7) imply that $\lim _{n \rightarrow \infty}\left\||T|^{\frac{1}{2}}(T-z)^{k-1} \bar{\partial}^{i} f_{n}\right\|_{2, D}=0$ for $i=$ $1, \cdots, 4 k-2$. Since $T=U|T|$, from (3) we have

$$
\lim _{n \rightarrow \infty}\left\|z(T-z)^{k-1} \bar{\partial}^{i} f_{n}\right\|_{2, D_{0}}=0
$$

for $i=1, \cdots, 4 k-2$. By an application of [14, Proposition 2.1] with $T=0$,

$$
\lim _{n \rightarrow \infty}\left\|(I-P)(T-z)^{k-1} \bar{\partial}^{i} f_{n}\right\|_{2, D_{0}}=0
$$

for $i=1, \cdots, 4 k-4$. From (8) and (9), we obtain

$$
\lim _{n \rightarrow \infty}\left\|z P(T-z)^{k-1} \bar{\partial}^{i} f_{n}\right\|_{2, D_{0}}=0
$$

for $i=1, \cdots, 4 k-4$. By $\underline{6}$, there exists a constant $c>0$ such that

$$
c\left\|P(T-z)^{k-1} \bar{\partial}^{i} f_{n}\right\|_{2, D_{0}} \leq\left\|z P(T-z)^{k-1} \bar{\partial}^{i} f_{n}\right\|_{2, D_{0}}
$$

for $i=1, \cdots, 4 k-4$. Hence from (10) we have $\lim _{n \rightarrow \infty}\left\|P(T-z)^{k-1} \bar{\partial}^{i} f_{n}\right\|_{2, D_{0}}=0$ for $i=1, \cdots, 4 k-4$. Then (9) implies that $\lim _{n \rightarrow \infty}\left\|(T-z)^{k-1} \bar{\partial}^{i} f_{n}\right\|_{2, D_{0}}=0$ for $i=1, \cdots, 4 k-4$. Thus $\lim _{n \rightarrow \infty}\left\|(T-z) \bar{\partial}^{i} f_{n}\right\|_{2, D_{0}}=0$ for $i=1, \cdots, 4$. In particular, if $k=1$ in $(9)$, we have $\lim _{n \rightarrow \infty}\left\|(I-P) f_{n}\right\|_{2, D_{0}}=0$. So we complete the proof.

Now we are ready to prove our main theorem.

Theorem 4.2. Let $T \in \mathcal{L}(H)$ be a p-hyponormal operator where $0<p \leq 1$. If $S \in$ Helton $_{k}(T)$, then $S$ is a subscalar operator of order $4 k$.

Proof. Consider an arbitrary bounded open disk $D$ of the complex plane $C$ containing $\sigma(S)$ and 0 and the quotient space

$$
H(D)=W^{4 k}(D, H) \overline{(z-S) W^{4 k}(D, H)}
$$

endowed with the Hilbert space norm. The class of a vector $f$ or an operator $A$ on $H(D)$ will be denoted by $\tilde{f}$, respectively $\tilde{A}$. Note that $M$, the multiplication operator with $z$ on $W^{4 k}(D, H)$, leaves invariant $\operatorname{ran}(z-S)$; hence $\tilde{M}$ is well defined. Moreover, the spectral distribution $\Phi$ of $M$ commutes with $z-S$; therefore, $\tilde{M}$ is still a scalar operator of order $4 k$, with $\tilde{\Phi}$ as spectral distribution.

Let $V$ be the operator $V(h)=\widetilde{1 \otimes h}$, from $H$ into $H(D)$, denoting by $1 \otimes h$ the constant function $h$. Then $V S=\tilde{M} V$. Indeed, $V S h=\widetilde{1 \otimes S h}=\widetilde{z \otimes h}=$ $\tilde{M}(\widetilde{1 \otimes h})=\tilde{M} V h$ for any $h \in H$. Since $\operatorname{ran} V$ is an invariant subspace for $\tilde{M}$, it suffices to show that $V$ is one-to-one and has closed range.

If $h_{n} \in H$ and $f_{n} \in W^{4 k}(D, H)$ are sequences such that

$$
\lim _{n \rightarrow \infty}\left\|(z-S) f_{n}+1 \otimes h_{n}\right\|_{W^{4 k}}=0,
$$


then we have

$$
\lim _{n \rightarrow \infty}\left\|(z-S) \bar{\partial}^{i} f_{n}\right\|_{2, D}=0
$$

for $i=1,2, \cdots, 4 k$. Now for $i=1,2, \cdots, 4 k$,

$$
\begin{aligned}
& \lim _{n \rightarrow \infty}\left\|\sum_{j=0}^{k}(-1)^{k-j}\left(\begin{array}{c}
k \\
j
\end{array}\right) T^{j} S^{k-j} \bar{\partial}^{i} f_{n}-(T-z)^{k} \bar{\partial}^{i} f_{n}\right\|_{2, D} \\
= & \lim _{n \rightarrow \infty}\left\|\sum_{j=0}^{k}\left(\begin{array}{c}
k \\
j
\end{array}\right)(T-z)^{j}(z-S)^{k-j} \bar{\partial}^{i} f_{n}-(T-z)^{k} \bar{\partial}^{i} f_{n}\right\|_{2, D} \\
= & \lim _{n \rightarrow \infty}\left\|\sum_{j=0}^{k-1}\left(\begin{array}{c}
k \\
j
\end{array}\right)(T-z)^{j}(z-S)^{k-j} \bar{\partial}^{i} f_{n}\right\|_{2, D} \\
\leq & \lim _{n \rightarrow \infty}\left\|\sum_{j=0}^{k-1}\left(\begin{array}{c}
k \\
j
\end{array}\right)(T-z)^{j}(z-S)^{k-j-1}\right\|\left\|(z-S) \bar{\partial}^{i} f_{n}\right\|_{2, D} \\
= & 0 .
\end{aligned}
$$

Hence we have $\lim _{n \rightarrow \infty}\left\|(T-z)^{k} \bar{\partial}^{i} f_{n}\right\|_{2, D}=0$ for $i=1,2, \cdots, 4 k$. Then Lemma 4.1 can be applied to get

$$
\lim _{n \rightarrow \infty}\left\|(I-P) f_{n}\right\|_{2, D_{0}}=0,
$$

where a disk $D_{0}$ is strictly contained in $D$. Now we apply (13) to (11). Then we get

$$
\lim _{n \rightarrow \infty}\left\|(z-S) P f_{n}+1 \otimes h_{n}\right\|_{2, D_{0}}=0 .
$$

Let $\Gamma$ be a curve in $D_{0}$ surrounding $\sigma(S)$. Then for $z \in \Gamma$,

$$
\lim _{n \rightarrow \infty}\left\|P f_{n}(z)+(z-S)^{-1}\left(1 \otimes h_{n}\right)\right\|=0
$$

uniformly. Hence by the Riesz-Dunford functional calculus we have

$$
\lim _{n \rightarrow \infty}\left\|\frac{1}{2 \pi i} \int_{\Gamma} P f_{n}(z) d z+h_{n}\right\|=0 .
$$

But since $\int_{\Gamma} P f_{n} d z=0$ by Cauchy's theorem, $\lim _{n \rightarrow \infty} h_{n}=0$. Hence $V$ is one-toone and has closed range.

Corollary 4.3. Every p-hyponormal operator is subscalar of order 4.

Proof. If $k=1$ in Theorem 4.2, then $S=T$. Hence the proof follows from Theorem 4.2 .

If $U$ is a bounded open set in the complex plane $\mathbf{C}$, recall that a subset $\Lambda \subset U$ is said to be dominating for $U$ if every function $h(z)$ analytic and bounded on $U$ satisfies

$$
\sup _{z \in U}|h(z)|=\sup _{z \in U \cap \Lambda}|h(z)| .
$$

Corollary 4.4. Let $S \in$ Helton $_{k}(T)$ where $T \in \mathcal{L}(H)$ is a p-hyponormal operator with $0<p \leq 1$. If there exists a nonempty open set $U$ in the complex plane $\mathbf{C}$ such that $\sigma(S) \cap U$ is dominating for $U$, then $S$ has a nontrivial invariant subspace.

Proof. This follows from Theorem 4.2 and [7]. 
From Theorem 4.2 we get the following corollary.

Corollary 4.5. If $S \in$ Helton $_{k}(T)$ where $T \in \mathcal{L}(H)$ is a p-hyponormal operator with $0<p \leq 1$, then

(a) $S$ has the property $(\beta)$;

(b) $S$ has the single-valued extension property.

Proof. Since every scalar operator satisfies the property $(\beta)$ and the property $(\beta)$ is transmitted from an operator to its restrictions to closed invariant subspaces, it follows from Theorem 4.2 that $S$ satisfies the property $(\beta)$. In particular, it has the single-valued extension property.

We close this paper with the following remark pointed out by the referee.

Remark. $T^{*}$ being in the Helton class of order $k$ of $T$ translates to (in Helton's terminology) that $T^{*}$ is coadjoint of order $k-1$, from which it follows (as in [9]) that $\left\|e^{i s T^{*}}\right\|=O\left(|s|^{k-1}\right)$. Hence $T^{*}$ (and also $T$ ) possesses a continuous $C^{k}(\mathbf{R})$ functional calculus. But then it follows that $T$ is decomposable (see [5]). By definition any decomposable operator having spectrum with more than one point has nontrivial invariant subspaces. Helton conjectured and proved in some special case while Agler provided a proof for the general case (at least for $k=3$ ) (see also the paper of Ball and Helton, 3]) an alternative characterization of coadjoint operators as sub-Jordan operators. Sub-Jordan operators appear to be related to but a priori different from subscalar operators.

\section{ACKNOWLEDGMENTS}

The authors would like to express their gratitude to the referee for valuable comments.

\section{REFERENCES}

[1] J. Agler, Sub-Jordan operators: Bishop's theorem, spectral inclusion, and spectral sets, J. Operator Theory $\mathbf{7}(1982), 373-395$. MR0658619 (83j:47009)

[2] A. Aluthge, On p-hyponormal operators for $0<p<1$, Int. Eq. Op. Th. 13(1990), 307-315. MR.1047771 (91a:47025)

[3] J.A. Ball and J.W. Helton, Nonnormal dilations, disconjugacy and constrained spectral factorization, Int. Eq. Op. Th. 3(1980), 216-309. MR0577164 (83a:47007)

[4] E. Bishop, A duality theorem for an arbitrary operator, Pacific J. Math. 9(1959), 379-397. MR0117562 (22:8339)

[5] I. Colojoara and C. Foias, Theory of generalized spectral operators, Gordon and Breach, New York, 1968. MR0394282 (52:15085)

[6] J.B. Conway, Subnormal operators, Pitman Adv. Pub. Program, 1981. MR0634507 (83i:47030)

[7] J. Eschmeier, Invariant subspaces for subscalar operators, Arch. Math. 52(1989), 562-570. MR1007631 (90h:47016)

[8] J.W. Helton, Operators with a representation as multiplication by $x$ on a Sobolev space, Colloquia Math. Soc. Janos Bolyai 5, Hilbert Space Operators, Tihany, Hungary (1970), 279-287. MR 0367687(51:3929)

[9] J.W. Helton, Infinite dimensional Jordan operators and Sturm-Liouville conjugate point theory, Trans. Amer. Math. Soc. 170(1972), 305-331. MR0308829 (46:7943)

[10] E. Ko, On p-hyponormal operators, Proc. Amer. Math. Soc. 128(2000), 775-780. MR.1707152 (2000e:47040)

[11] E. Ko, w-Hyponormal operators have scalar extensions, Int. Eq. Op. Th. 53(2005), 363-372. MR2186096 (2006g:47033) 
[12] R. Lange and S. Wang, New approaches in spectral decomposition, Contemporary Math. 128, Amer. Math. Soc., 1992. MR.1162741 (93i:47039)

[13] K. Löwner, Über monotone matrix funktionen, Math. Z. 38(1934), 177-216.

[14] M. Putinar, Hyponormal operators are subscalar, J. Operator Theory 12(1984), 385-395. MR0757441 (85h:47027)

[15] D. Xia, Spectral theory of hyponormal operators, Op. Th.: Adv. Appl. 10, Birkhäuser Verlag, Basel, 1983. MR0806959(87j:47036)

Department of Mathematics, Ewha Women's University, Seoul 120-750, Korea

E-mail address: yoenha@ewhain.net

Department of Mathematics, Ewha Women's University, Seoul 120-750, Korea

E-mail address: eiko@ewha.ac.kr

Department of Mathematics, Ewha Women's University, Seoul 120-750, Korea

E-mail address: jieun7@ewhain.net 